Received: 08.07.2018

Revised: 10.09.2018

Accepted: 26.10 .2018

DOI: $10.17804 / 2410-9908.2018 .5 .116-126$

\title{
SPECIAL CHARACTERISTICS OF THE MICROSTRUCTURE OF HIGH-STRENGTH MULTIFILAMENTARY Cu-18Nb COMPOSITES
}

\author{
E. G. Valova-Zaharevskaya ${ }^{1, \text { a })^{*}}$, I. L. Deryagina ${ }^{1, b)}$, E. N. Popova ${ }^{1, c)}$, \\ N. E. Khlebova, d), and V. I. Pantsyrny ${ }^{2, \text { e) }}$ \\ ${ }^{1}$ M.N. Miheev Institute of Metal Physics, Ural Branch of the Russian Academy of Sciences, \\ 18 S. Kovalevskoy St., Ekaterinburg, 620108, Russian Federation \\ ${ }^{2}$ Nanoelectro Research and Production Company, \\ 5 A Rogova St., Moscow, Russian Federation \\ a) (iD https://orcid.org/0000-0002-9206-4727; valova@imp.uran.ru;

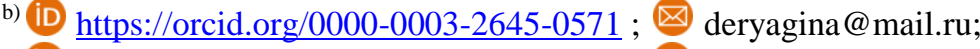 \\ c) (ID https://orcid.org/0000-0002-4814-4420 ; popova@imp.uran.ru; \\ d) khlebna@gmail.com; \\ e) (iD https://orcid.org/0000-0002-1974-9148 ; ه pantsyrny @ gmail.com \\ *Corresponding author. E-mail: valova@imp.uran.ru \\ Address for correspondence: ul. S. Kovalevskoy, 18, Ekaterinburg, 620108, Russian Federation \\ Tel.: +7 (343) 37835 41; fax: +7 (343) 3745244
}

The paper presents refined data on the macroscopic and microscopic stresses according to $\mathrm{X}$-ray diffraction analysis in multifilamentary $\mathrm{Cu}-18 \mathrm{Nb}$ composites fabricated by the melt-anddeform (in-situ) route, with a true strain $e$ of 10.2 and 12.5. Under large plastic deformation by cold drawing, $\mathrm{Nb}$ dendrites in the $\mathrm{Cu}$ matrix acquire the shape of ribbons with the thickness decreasing to below $100 \mathrm{~nm}$ with increasing strain. The sharp fiber texture $\langle 110\rangle \mathrm{Nb} \|<111\rangle \mathrm{Cu} \|$ DA (deformation axis) develops in the composites, and its degree grows with increasing strain. The $\mathrm{Nb}$ crystal lattice is distorted due to the partially coherent character of the $\mathrm{Cu} / \mathrm{Nb}$ interfaces, and these distortions also increase with strain. They are manifested in $\{110\}_{\mathrm{Nb}}$ interplanar spacings extended in the longitudinal sections of the composites and compressed in the cross sections. In the $\mathrm{Cu}$ matrix lattice distortions are also observed, though they are much less pronounced, this being attributable to the dynamic recrystallization of copper and the weakening of its texture, even when in the composite.

Keywords: high-strength composites, $\mathrm{Cu}-\mathrm{Nb}$, lattice parameters, X-ray diffraction analysis.

\section{Acknowledgment}

The work was performed on the equipment of the collective use center of IPM UB RAS within the state assignment from FASO Russia (theme Pressure, No. AAAA-A18-118020190104-3), with a partial support from UB RAS (project No. 18-10-2-24).

\section{References}

1. Heringhaus F., Raabe D. Recent Advances in the Manufacturing of Copper-Base Composites. J. Mater. Proc. Technol., 1996, vol. 59, pp. 367-372. DOI: 10.1016/0924-0136(95)02179-5.

2. Raabe D., Mattissen D. Microstructure and mechanical properties of a cast and wiredrawn ternary $\mathrm{Cu}-\mathrm{Ag}-\mathrm{Nb}$ in situ composite. Acta mater., 1998, vol. 46, no. 16, pp. 5973-5984. DOI: $10.1016 /$ S1359-6454(98)00218-3. 
3. Han K., Embury J.D., Sims J.R., Campbell L.J., Schneider-Muntau H.-J., Pantsyrnyi V.I., Shikov A., Nikulin A., Vorobieva A. The fabrication, properties and microstructure of $\mathrm{Cu}-\mathrm{Ag}$ and $\mathrm{Cu}-\mathrm{Nb}$ composites. Mater. Sci. Eng., 1999, vol. A267, pp. 99-114. DOI: 10.1016/S09215093(99)00025-8.

4. Han K., Lawson A.C., Wood J.T., Embury J.D., Von Dreele R.B., Richardson J.W. Internal stresses in cold-deformed $\mathrm{Cu}-\mathrm{Ag}$ and $\mathrm{Cu}-\mathrm{Nb}$ wires. Phil. Mag., 2004, vol. 84, no. 24, pp. 2579-2593. DOI: 10.1080/14786430410001689981.

5. Zuo X., Zhu J., An B., Han K., Li R., Wang E. Influence of Fe addition on microstructure and properties of $\mathrm{Cu}-\mathrm{Ag}$ composite. Met. Mater. Int., 2017, vol. 23, no. 5, pp. 974-983. DOI: $10.1007 / \mathrm{s} 12540-017-6656-2$.

6. Bevk J., Harbison J.P., Bell J.L. Anomalous increase in strength of in situ formed Cu-Nb multifilamentary composites. J. Appl. Phys., 1978, vol. 49, no. 12, pp. 6031-6038. DOI: $10.1063 / 1.324573$.

7. Spitzig W.A., Krotz P.D. A comparison of the strength and microstructure of heavily cold worked $\mathrm{Cu}-20 \% \mathrm{Nb}$ composite formed by different melting procedures. Scripta Metall., 1987, vol. 21, no. 8, pp. 1143-1146. DOI: 10.1016/0036-9748(87)90266-3.

8. Shikov A., Pantsyrnyi V., Vorobieva A., Khlebova N., Silaev A. High strength, high conductivity $\mathrm{Cu}-\mathrm{Nb}$ based conductors with nanoscaled microstructure. Physica C: Superconductivity, 2001, vol. 354, no. 1-4, pp. 410-414. DOI: 10.1016/S0921-4534(01)00109-5.

9. Pantsyrnyi V. Status and perspectives for microcomposite winding materials for high field pulsed magnets. IEEE Trans. Appl. Supercond., 2002, vol. 12, iss. 1, pp. 1189-1194. DOI: $10.1109 /$ TASC.2002.1018614.

10. Pantsyrny V., Shikov A., Vorobieva A., Khlebova N., Kozlenkova N., Potapenko I., Polikarpova M. Stability Aspects of the High Strength High Conductivity Microcomposite $\mathrm{Cu}-\mathrm{Nb}$ Wires Properties. IEEE Trans. Appl. Supercond., 2006, vol. 16, no. 2, pp. 1656-1659. DOI: 10.1109/TASC.2006.870554.

11. Leprince-Wang Y., Han K., Huang Y., Yu-Zhang K. Microstructure in $\mathrm{Cu}-\mathrm{Nb}$ microcomposites. Mater. Sci. Eng. A, 2003, vol. 351, iss. 1-2, pp. 214-223. DOI: 10.1016/S0921-5093(02)00855-9.

12. Nishijima G., Awaji S., Watanabe K., Miyoshi K., Kimura A. Transport characteristics of a $\mathrm{CuNb} / \mathrm{Nb}_{3} \mathrm{Sn}$ superconducting coil fabricated using a react and wind method. Supercond. Sci. Technol., 2003, vol. 16, pp. 1082-1085. DOI: 10.1088/0953-2048/16/9/320.

13. Sandim M.J.R., Stamopoulos D., Aristomenopoulou E., Zaefferer S., Raabe D., Awaji S., Watanabe K. Grain structure and irreversibility line of a bronze route $\mathrm{CuNb}$ reinforced $\mathrm{Nb}_{3} \mathrm{Sn}$ multifilamentary wire. Phys. Procedia, 2012, vol. 36, pp. 1504-1509. DOI: 10.1016/j.phpro.2012.06.122.

14. Oguro H., Awaji S., Watanabe K., Sugimoto M., Tsubouchi H. Prebending Effect for Mechanical and Superconducting Properties of Nb-Rod-Processed $\mathrm{Cu}-\mathrm{Nb}$ Internal-Reinforced $\mathrm{Nb}_{3} \mathrm{Sn}$ Wires. IEEE Transact. Appl. Supercond., 2014, vol. 24, no. 3, pp. 8401004 (4). DOI: 10.1109/tasc.2013.2292507.

15. Sugimoto M., Tsubouchi H., Endoh S., Takagi A., Watanabe K., Awaji S., Oguro H. Development of Nb-Rod-Method $\mathrm{Cu}-\mathrm{Nb}$ Reinforced Nb3Sn Rutherford Cables for React-and-Wind Processed Wide-Bore High Magnetic Field Coils. Transact. IEEE Transactions on Applied Superconductivity, 2015, vol. 25, iss. 3, pp. 6000605. DOI: 10.1109/TASC.2014.2381459.

16. Popova E.N., Popov V.V., Rodionova L.A., Sudareva S.V., Romanova E.P., Vorobyova A.E., Hlebova N.E., Pantsyrnyi V.I., Shikov A.K. Microstructure and texture of in situ heavily drawn $\mathrm{Cu}-\mathrm{Nb}$ composites. Textures and Microstructures, 2000, vol. 34, pp. 263-277. DOI: $10.1155 /$ TSM.34.263.

17. Popova E.N., Popov V.V., Romanov E.P., Rodionova L.A., Sudareva S.V., Khlebova N.E., Pantsyrnyi V.I., Shikov A.K., Vorob'eva A.E. Effect of alloying and regimes of thermo-mechanical treatment on the structure and properties of $\mathrm{Cu}-\mathrm{Nb}$ composites. Phys. Met. Metallogr., 2002, vol. 94, no. 1, pp. 73-81. 
18. Deryagina I.L., Popova E.N., Valova-Zaharevskaya E.G., Patrakov E.I. Structure and Thermal Stability of High-Strength $\mathrm{Cu}-18 \mathrm{Nb}$ Composite Depending on the Degree of Deformation. Phys. Met. Metallogr., 2018, vol. 119, no. 1, pp. 92-102. DOI: 10.1134/S0031918X18010088.

19. Deng L., Yang X., Han K., Lu Y., Liang M., Liu Q. Microstructure and texture evolution of $\mathrm{Cu}-\mathrm{Nb}$ composite wires. Mater. Character., 2013, vol 81, pp. 124-133. DOI: 10.1016/j.matchar.2013.04.013.

20. Deng L., Han K., Hartwig K.T., Siegrist T.M., Dong L., Sun Z., Yang X., Liu Q. Hardness, electrical resistivity, and modeling of in situ $\mathrm{Cu}-\mathrm{Nb}$ microcomposites. J. Alloys and Compounds, 2014, vol. 602, pp. 331-338. DOI: 10.1016/j.jallcom.2014.03.021.

21. Sauvage X., Renaud L., Deconihout B., Blavette D., Ping D., Hono H. Solid state amorphization in cold drawn $\mathrm{Cu} / \mathrm{Nb}$ wires. Acta Mater., 2001, vol. 49, pp. 389-394. DOI: $10.1016 / \mathrm{S} 1359-6454(00) 00338-4$.

22. Dupouy F., Snoeck E., Casanove M.J., Roucau C., Peyrade J.P., Askenazy S. Microstructural characterization of high strength and high conductivity nanocomposite wires. Scripta Mater., 1996, vol. 34, no. 7, pp. 1067-1073. DOI: 10.1016/1359-6462(95)00632-X.

23. Snoeck E., Lecouturier F., Thilly L., Casanove M.J., Rakoto H., Coffe G., Aske'nazy S., Peyrade J.P., Roucau C., Pantsyrny V., Shikov A., Nikulin A. Microstructural studies of in situ produced filamentary $\mathrm{Cu} / \mathrm{Nb}$ wires. Scripta Mater., 1998, vol. 38, no. 11, pp. 1643-1648. DOI: 10.1016/S1359-6462(98)00080-3.

24. Hangen U., Raabe D. Modelling of the yield strength of a heavily wire drawn $\mathrm{Cu}-20 \% \mathrm{Nb}$ composite by use of a modified linear rule of mixtures. Acta Metall. Mater., 1995, vol. 43, no. 11, pp. 4075-4082. DOI: 10.1016/0956-7151(95)00079-B.

25. Pelton A.R., Laabs F.C., Spitzig W.A., Chen C.C. Microstructural analysis of in-situ copperniobium composite wires. Ultramicroscopy, 1987, vol. 22 (1-4), pp. 251-265. DOI: 10.1016/03043991(87)90069-6.

26. Kalu P.T., Brandao L., Ortiz F., Okechukwu Egungwu, Funmi Ige. On the texture evolution in swaged Cu-based wires. Scripta Mater., 1998, vol. 38, no. 12, pp. 1755-1761. DOI: $10.1016 / \mathrm{S} 1359-6462(98) 00114-6$.

27. Shikov A.K., Pantsyrnyi V.I., Vorob'eva A.E., Sud'ev S.V., Khlebova N.E., Silaev A.K., Belyakov N.A. Copper-niobium high-strength and high-conductivity winding wires for pulsed magnets. Metal Science and Heat Treatment, 2002, vol. 44, pp. 491-495. DOI: 10.1023/A:1022504805662.

28. Yu-Zhang K., Embury J.D., Han K., Misra A. Transmission electron microscopy investigation of the atomic structure of interfaces in nanoscale $\mathrm{Cu}-\mathrm{Nb}$ multilayers. Phil. Mag., 2008, vol. 88, no. 17 , pp. 2559-2567. DOI: 10.1080/14786430802380485. 
Подана в журнал: 08.07.2018

УДК 669.15'26’27'292-194:539.25

DOI: $10.17804 / 2410-9908.2018 .5 .116-126$

\title{
ОСОБЕННОСТИ МИКРОСТРУКТУРЫ ВЫСОКОПРОЧНОГО МНОГОВОЛОКОННОГО КОМПОЗИТА $\mathrm{Cu}-18 \mathrm{Nb}$
}

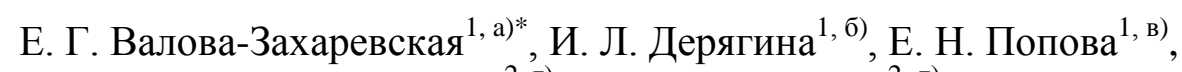 \\ Н. Е. Хлебова ${ }^{2, \text { г) }}$ В. И. Панцырный ${ }^{2, \text { д) }}$
}

${ }^{1}$ Институт физики металлов им. М.Н. Михеева Уральского отделения Российской академии наук,
620108, ул. С. Ковалевской, 18, г. Екатеринбург, Российская Федераџия
${ }^{2}$ Научно-производственное предприятие «НАНОЭЛЕКТРО»,
123098, ул. Рогова, 5А, г. Москва, Российская Федерация

a) iD https://orcid.org/0000-0002-9206-4727 ; valova@imp.uran.ru;

б) (iD https://orcid.org/0000-0003-2645-0571 ; ه deryagina@mail.ru ;

в) iD https://orcid.org/0000-0002-4814-4420 ; popova@imp.uran.ru ;

г) $\otimes$ khlebna@gmail.com ;

д) iD https://orcid.org/0000-0002-1974-9148 ; ه pantsyrny@ gmail.com ;

*Ответственный автор. E-mail: valova@imp.uran.ru

Адрес для переписки: ул. С. Ковалевской, 18, 620108, Екатеринбург, Российская Федерация

Тел: +7 (343) 378-35-41; факс: +7 (343) 374-52-44

В работе представлены уточненные данные расчетов макро- и микронапряжений по данным рентгеноструктурного анализа в многоволоконных композитах $\mathrm{Cu}-18 \mathrm{Nb}$, полученных методом плавка-деформация (in-situ), со степенью истинной деформации (e) 10,2 и 12,5. В процессе сильной пластической деформации многократным холодным волочением дендриты ниобия в медной матрице приобретают форму лент, толщина которых с ростом степени деформации становится меньше 100 нм. При этом в композите развивается острая аксиальная текстура $<110>\mathrm{Nb}\|<111>\mathrm{Cu}\|$ ОД (оси деформации), степень которой растет с увеличением деформации. В кристаллической решетке ниобия обнаружены искажения, обусловленные полукогерентным характером границ раздела $\mathrm{Nb}$ лент и медной матрицы, которые также растут с увеличением степени деформации. Эти искажения проявляются в том, что межплоскостные расстояния $\{110\}_{\mathrm{Nb}}$ в продольном сечении композитов растянуты, а в поперечном сжаты. Обнаружены и искажения решетки медной матрицы, хотя они выражены значительно в меньшей степени, что может быть обусловлено динамической рекристаллизацией меди и ослаблением ее текстуры даже в условиях композита.

Ключевые слова: высокопрочные композиты, $\mathrm{Cu}-\mathrm{Nb}$, параметры кристаллической решетки, рентгеноструктурный анализ.

\section{1. Введение}

Микрокомпозиты на основе меди сочетают высокую прочность с высокой электропроводностью, что делает этот класс материалов крайне перспективным в электрических устройствах с высокой механической нагрузкой, в частности в устройствах для получения сильных магнитных полей. В качестве добавок к медной матрице в большинстве случаев используют ОЦК переходные металлы, обладающие крайне низкой растворимостью в меди $(\mathrm{Nb}, \mathrm{Fe}, \mathrm{Cr}, \mathrm{V})$ или ГЦК Ag [1-5].

Композиционные проводники, в которых медная матрица упрочняется тонкими ниобиевыми волокнами, занимают особое место среди всех in situ композитов, и им посвящается наибольшее количество публикаций, начиная от конца прошлого столетия и до наших дней 
[6-11]. Это обусловлено тем, что проводники системы $\mathrm{Cu}-\mathrm{Nb}$ демонстрируют наивысшие значения временного сопротивления разрыву $\left(\sigma_{\mathrm{B}}\right)$, вплоть до 2000-2400 МПа при 18-20\% ниобия. Эффект аномального повышения прочности начинает проявляться при истинной деформации выше 5, когда размер структурных составляющих становится меньше 100 нм, т. е. становится соизмеримым с характеристическими размерными параметрами многих физических величин (свободный пробег электронов, протяженность дислокаций и дисклинаций, размер доменов в ферромагнетиках и др.).

Наряду с высокой прочностью микрокомпозиты $\mathrm{Cu}-18 \mathrm{Nb}$ могут обладать высокой электропроводностью (порядка $70 \%$ от электропроводности чистой меди по международным стандартам IACS - International Annealed Copper Standard, где $100 \%$ IACS = 1,724 $\mathrm{m} \Omega \cdot \mathrm{cm})$, и поэтому они перспективны для использования в электромагнитах с высокими (вплоть до 100 Тл) полями, в ЯМР приборах с полями от 15 до 20 Тл и на участках электросетей, подвергающихся экстремальным механическим нагрузкам [9]. Кроме того, эти композиты представляют большой интерес для упрочнения сверхпроводников на основе $\mathrm{Nb}_{3} \mathrm{Sn}$ [12-15].

На протяжении многих лет различные конструкции проводов на основе микрокомпозитов $\mathrm{Cu}-\mathrm{Nb}$ разрабатывались во ВНИИНМ им. ак. А.А. Бочвара (Москва, Россия) [8-10]. Первые провода микрокомпозитов $\mathrm{Cu}-\mathrm{Nb}$ изготавливались в виде тонкой, так называемой одноволоконной in-situ проволоки. Особенности их структуры, текстуры и возможные механизмы упрочнения изучены и проанализированы в целом ряде наших ранних работ $[16,17]$.

Позднее во ВНИИНМ была разработана конструкция высокопрочных многоволоконных проводов различной формы с поперечными размерами до десятка миллиметров на основе микрокомпозита $\mathrm{Cu}-18 \mathrm{Nb}$ [10]. Образцы одного из таких проводов с различной величиной деформации исследованы в настоящей работе.

Цель работы - уточнение особенностей изменений кристаллической решетки ниобиевой и медной фазы в массивных многоволоконных композитах $\mathrm{Cu}-18 \mathrm{Nb}$ с разной степенью истинной деформации.

\section{2. Материалы и методика}

Образцы многоволоконного микрокомпозита $\mathrm{Cu}-18 \mathrm{Nb}$ после истинной деформации $e=10,2$ (образец 1 - цилиндрической формы, диаметр 15,4 мм) и $e=12,5$ (образец $2-$ прямоугольной формы, размеры $3 \times 5,8$ мм) были разработаны и изготовлены в АО ВНИИНМ [10]. Основу конструкции исследованных в работе композитов составляли 600 in situ одноволоконных стрендов $\mathrm{Cu}-18 \mathrm{Nb}$, размещенных в матрице из высокочистой меди.

Подробное исследование микроструктуры и состава микрокомпозитов методами сканирующей и просвечивающей электронной микроскопии, а также данные о микротвердости исследуемых образцов представлены в [18].

Степень текстуры, изменение параметра решетки меди и ниобия оценивали по результатам рентгеноструктурного анализа (на аппарате ДРОН, с монохроматором и фиксированной щелью в излучении $\mathrm{Cr}\left(\mathrm{K}_{\alpha 1}+\mathrm{K}_{\alpha 2}\right)$ в диапазоне углов $25 \leq 2 \Theta \leq 140$ град.) на продольных и поперечных шлифах образцов в исходном состоянии и после отжига в вакууме при $800{ }^{\circ} \mathrm{C}$, 1 ч. В настоящей работе первичную обработку, оценку и расчет отдельных пиков спектра проводили с помощью пакета программ для численного анализа данных Origin следующим образом. Для каждого образца из полученного спектра выделялся диапазон значений, соответствующий данному пику: пик 110 для ниобия, располагающийся в диапазоне углов от 56 до 62 град., а также пик 111 для меди, располагающийся в диапазоне углов от 66 до 67,5 град. Далее строили линейный график по выделенным значениям. При зашумленности пика производилось сглаживание спектральной линии путем фурье-преобразования. Коэффициент сглаживания функции выбирался наименьший, достаточный для получения гладкой функции без дополнительных максимумов и большого количества перегибов. Дальнейшая 
оценка и подгонка пика происходила на сглаженной линии пика. При аппроксимации пика была выбрана функция Гауссиан, с учетом физических представлений рассеяния рентгеновского излучения. По каждому пику получены параметры подгонки, соответствующие статистическим критериям. Формулы, используемые для расчетов параметров кристаллической решетки в исследуемых композитах по данным рентгеноструктурного анализа, также приведены в [18].

\section{3. Результаты эксперимента и их обсуждение}

При многоступенчатом волочении и сборке деформация распределяется по поперечным сечениям композитов неравномерно [18]. Основной же особенностью микроструктуры исследуемых образцов является сложная морфология изогнутых ниобиевых волокон ленточной формы (рис. $1 a$, б). Она отмечалась в многочисленных публикациях, и приписывается особенностям систем скольжения в ОЦК $\mathrm{Nb}$ и влиянию ГЦК медной матрицы $[3,6,8,19,20]$.

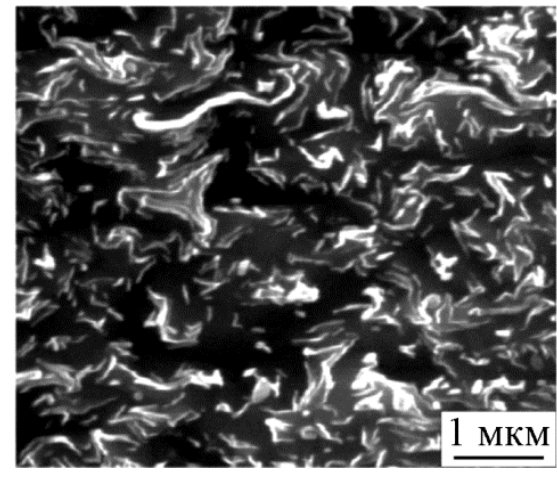

$a$

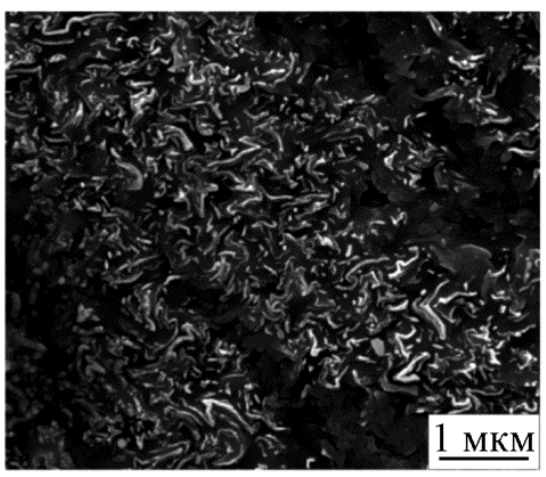

6

Рис. 1. Поперечные сечения образцов $1(a)$ и 2 (б) композита $\mathrm{Cu}-18 \mathrm{Nb}$

Уменьшение толщины ниобиевых лент и расстояния между ними с ростом деформации приводит к росту площади поверхности раздела между ленточным ниобием и зернами медной матрицы, а это, как показано в ряде работ по влиянию микроструктуры $\mathrm{Cu}-\mathrm{Nb}$ композитов на их свойства $[2-4,8,20]$, вызывает рост микротвердости и предела прочности. Действительно, микротвердость в исходном состоянии (до отжига) увеличивается с ростом степени деформации от 2400 МПа в образце $1(e=10,2)$ до $3300 \mathrm{MПа-}$ в образце $2(e=12,5)$ [18].

На отдельных электронограммах рефлексы, соответствующие $(110)_{\mathrm{Nb}}$, образуют гало диффузного рассеяния (указано стрелкой; рис. 2 б), что может косвенно свидетельствовать о наличии в ниобии на границе раздела с медной матрицей аморфной фазы, которая наблюдалась и другими авторами [21]. Кроме того, отметим, что при расчете большого количества электронограмм обоих образцов и с продольных, и с поперечных сечений было обнаружено, что межплоскостные расстояния (110) Nb меняются в достаточно широких пределах (от 2,30 до 2,40 нм при табличном значении 2,33 нм). Безусловно, точность расчета электронограмм невелика, но тем не менее можно заключить, что на отдельных участках имеются искажения решетки ниобия и в этих направлениях она либо растянута, либо сжата. Изложенные ниже результаты значительно более точного рентгеноструктурного анализа подтверждают этот вывод. 


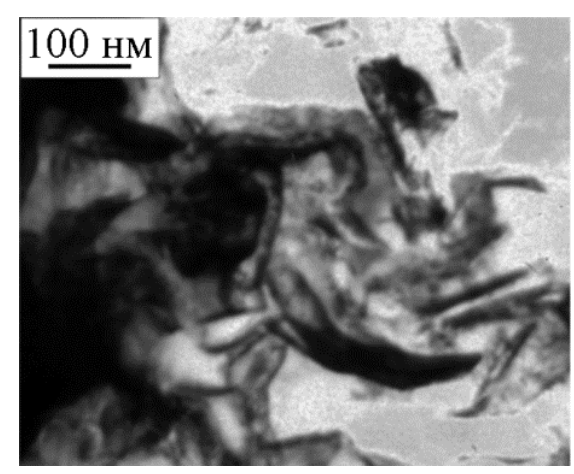

$a$

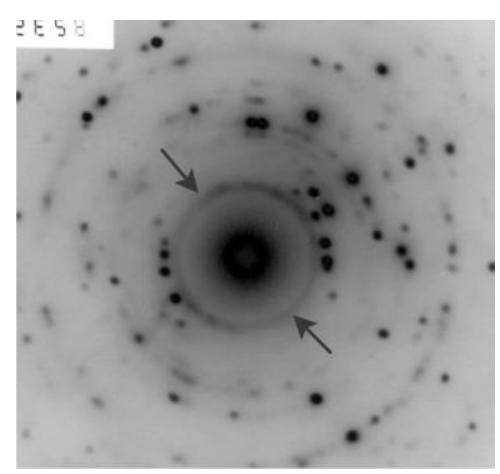

6

Рис. 2. Структура поперечного сечения композита $\mathrm{Cu}-18 \mathrm{Nb}$, образец 2 : $a$ - светлое поле; $\sigma$ - электронограмма (стрелками отмечено гало диффузионного рассеяния)

Для оценки степени текстуры и наличия искажений решетки ниобия в составе композита был проведен рентгеноструктурный анализ (PCA) образцов. В настоящей работе приведены результаты расчета при определении угла $2 \theta$ как положения центра массы пика. В случае ассиметрии положение максимума ассиметричного пика не совпадает с центром массы пика, что сопровождается отклонением от подгонки профиля пика гауссианом. В предыдущих расчетах [18] значения межплоскостного расстояния были получены исходя из визуального определения максимума пика, без учета его ассиметрии. В качестве эталонов нетекстурированного и ненапряженного состояния использовались данные РСА для изотропной порошковой меди и волокон ленточного ниобия, выделенного из состава образцов после растворения и удаления медной матрицы композита.

Данные рентгеноструктурного анализа показывают наличие в образцах 1 и 2 острой аксиальной текстуры $<110>_{\mathrm{Nb}}\left\|<111>_{\mathrm{Cu}}\right\|$ ОД (оси деформации), что согласуется с многочисленными данными многих более ранних исследований [16, 17, 22-27]. Кроме основной текстуры в меди наблюдается дополнительная текстура $<200>_{\text {си }} \|$ ОД. Она более слабая, и многие авторы не обнаруживали ее, хотя в ряде работ она также была отмечена $[16,25,26]$. С ростом степени деформации от 10,2 до 12,5 вес пика (111) Си увеличивается от 1,8 до 2,0, а пика $(110)_{\mathrm{Nb}}$ - от 1,9 до 2,1 , что свидетельствует о синхронном росте текстуры и в медной матрице, и в ниобии. В процентном отношении степень текстуры меди увеличивается с 84 до 94 \%, а ниобия - с 91 до 99 \% (табл. 1).

Таблица 1 - Структурные характеристики образцов на основе данных РСА

\begin{tabular}{|c|c|c|c|c|c|c|c|c|c|c|}
\hline $\begin{array}{c}\text { Обра- } \\
\text { зец }\end{array}$ & $e$ & $\begin{array}{c}\text { Сечение } \\
\text { провода }\end{array}$ & $\begin{array}{c}P_{111 \mathrm{Cu}}, \\
\%\end{array}$ & $\begin{array}{c}P_{110 \mathrm{Nb}}, \\
\%\end{array}$ & $\begin{array}{c}\text { ПШРП } \\
(111)_{\mathrm{Cu}}, \\
\circ\end{array}$ & $\begin{array}{c}\text { ПШРП } \\
(110)_{\mathrm{Nb}}, \\
\circ\end{array}$ & $\begin{array}{c}d_{110 \mathrm{Nb}} \\
\AA\end{array}$ & $\begin{array}{c}\Delta d_{110 \mathrm{Nb}}, \\
\%\end{array}$ & $\begin{array}{c}d_{111 \mathrm{Cu}} \\
\AA\end{array}$ & $\begin{array}{c}\Delta d_{111 \mathrm{Cu}}, \\
\%\end{array}$ \\
\hline 1 & 10,2 & Поперечное & 84,0 & 91 & 0,34 & 1,2 & 2,355 & $-0,85$ & 2,089 & $-0,16$ \\
\hline 1 & 10,2 & Продольное & & & & & 2,320 & 0,64 & 2,086 & 0,02 \\
\hline 2 & 12,5 & Поперечное & 94,0 & 99 & 0,46 & 1,5 & 2,380 & $-1,90$ & 2,082 & 0,17 \\
\hline 2 & 12,5 & Продольное & & & & & 2,288 & 2,02 & 2,088 & $-0,11$ \\
\hline $\mathrm{Nb}_{\text {эт }}$ & - & - & & & - & 0,5 & 2,335 & & - & \\
\hline $\mathrm{Cu}_{\text {эт }}$ & - & - & & & 0,24 & - & - & & 2,086 & \\
\hline
\end{tabular}

По изменению полуширины рентгеновских пиков (параметр ПШРП) была проведена качественная оценка изменения микронапряжений в меди и ниобии в составе композита.

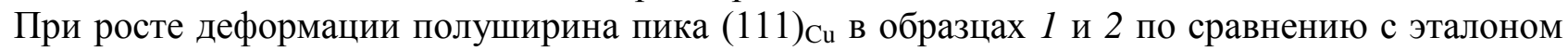
возрастает в 1,4 и 1,9 раза соответственно, а пика $(110)_{\mathrm{Nb}}$ по сравнению с эталоном - 
в 2,2 и 2,8 раза. Таким образом, в медной матрице и в волокнах ниобия композита существуют микронапряжения, которые возрастают с ростом деформации. При этом уровень микронапряжений в $\mathrm{Nb}$-волокнах выше, чем в медной матрице.

Помимо заметного уширения рентгеновских линий, которое мы приписываем присутствию внутренних напряжений в обоих составляющих композита, было обнаружено также смещение пиков, особенно ниобия, относительно эталонных значений (рис. 3).

Смещения пиков указывают на наличие искажений решетки. Смещение максимума $(110)_{\mathrm{Nb}}$ зависит как от плоскости шлифа, так и от степени деформации. На дифрактограммах от поперечных сечений (шлифов) пик (110) сечений (шлифов) - в сторону больших брэгговских углов (рис. $3 a$ ), причем с ростом деформации эти смещения пика увеличиваются.

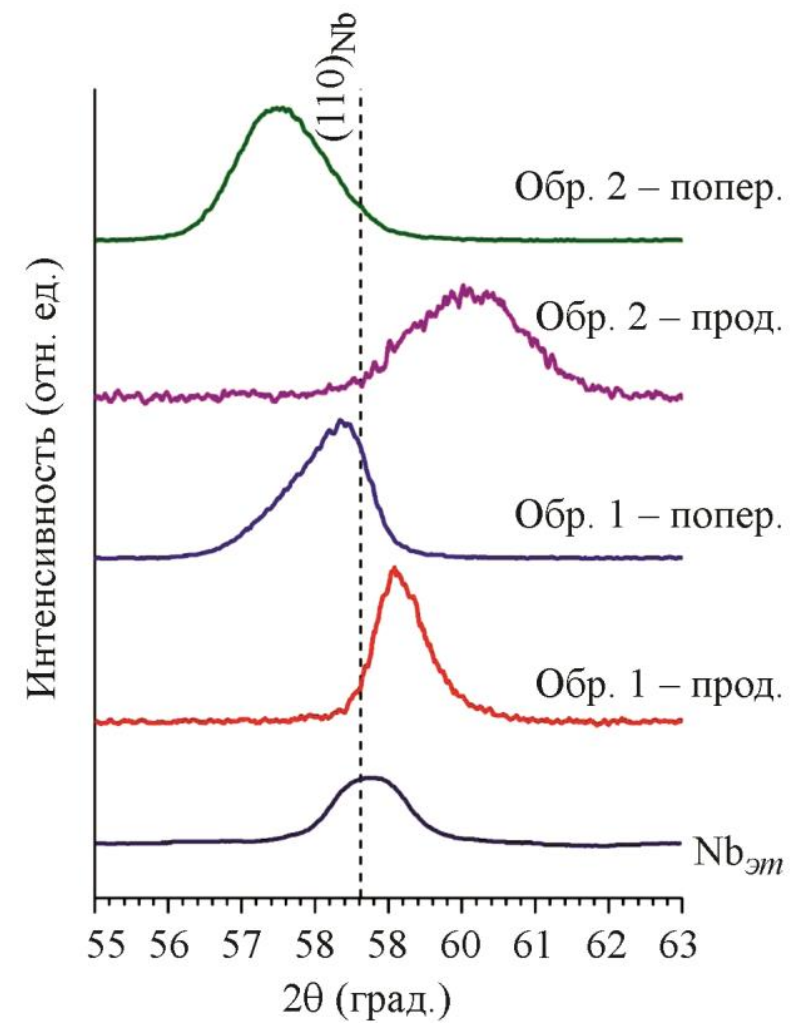

$a$

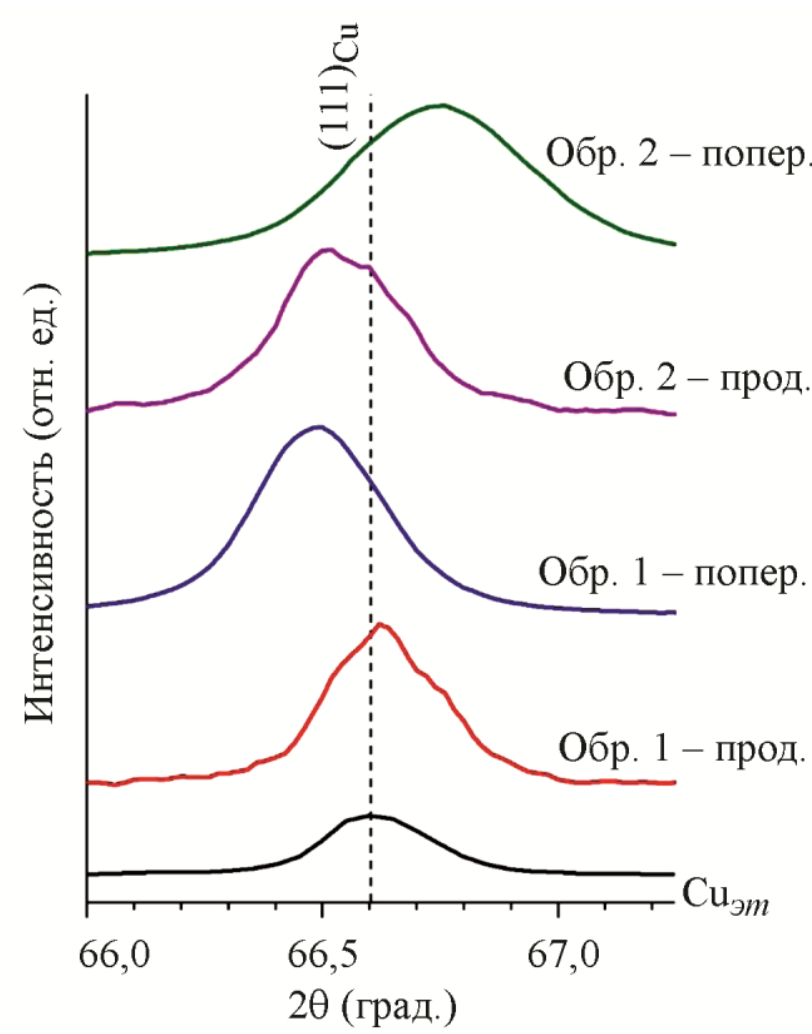

$\sigma$

Рис. 3. Положение пиков $(110)_{\mathrm{Nb}}(a)$ и $(111)_{\mathrm{Cu}}($ б) в деформированных образцах и эталонах

Таким образом, кристаллическая решетка ниобиевых волокон в матрице микрокомпозита искажена, и это искажение максимально в направлении, совпадающем с направлением текстуры ниобия $<110>_{\mathrm{Nb}}$. Межплоскостные расстояния $d_{110}$ ниобия растягиваются вдоль оси деформации и сжимаются в поперечном направлении. При увеличении деформации от 10,2 до 12,5 искажение межплоскостного расстояния (110) $\mathrm{Nb}$, как в продольном, так и в поперечном направлении провода, возрастает более чем в 3 раза (табл. 1).

Что касается искажений решетки меди, то они выражены значительно в меньшей степени и составляют десятые доли процента. При этом следует отметить две особенности. Вопервых, как и в ниобии, искажения несколько увеличиваются с ростом степени деформации, что, в принципе, вполне предсказуемо. Однако в отличие от ниобия, в меди не соблюдается закономерность в растяжении/сжатии решетки в зависимости от направления сечения (продольное или поперечное). Можно полагать, что это обусловлено несколькими факторами. Во-первых, это отсутствие ярко выраженной ленточной формы, как у ниобия. Во-вторых, как 
отмечено выше, в меди возможна и вторая компонента текстуры, которая может ослабляться с ростом степени деформации $[16,26]$. В целом, поскольку искажения в меди столь малы, можно полагать, что искажены лишь малые приграничные области, а во всем теле зерна решетка не искажена, и эти приграничные области могут быть по-разному искажены относительно оси деформации.

Несмотря на то, что разные методы расчета угла $2 \theta$ (положение центра массы пика и положение точки перегиба сглаженной функции) дают принципиально похожие результаты и близки данным, представленным в статье [18], применение первой методики в настоящей работе позволяет учесть ассиметрию пиков для лучшего определения изменений межплоскостных расстояний. Следует отметить, что полученные результаты об искажениях решеток ниобия и меди в таких композитах согласуются с результатами других авторов [8, 11 , $27,28]$. Так, например, согласно [27], в композите $\mathrm{Cu}-18 \mathrm{Nb}$ в интервале истинных деформаций $8,8>e>9,6$ возникают сильные искажения решетки ниобия $(110)_{\mathrm{Nb}}$ (сжатие в направлении, перпендикулярном оси волочения и растяжение параллельно этой оси), приводящие к увеличению степени несоответствия между решетками $(110)_{\mathrm{Nb}}$ и $(111)_{\mathrm{Cu}}$ с ростом степени деформации. При этом возрастает прочность композита, и возникают области когерентного сопряжения решеток меди и ниобия. В работе [11] искажения решеток обнаружены и в ниобии, и в меди, и авторы этой работы полагают, что наряду с большой площадью поверхностей раздела $\mathrm{Cu} / \mathrm{Nb}$ эти искажения вносят дополнительный вклад в упрочнение. В [28] большие искажения решеток и меди, и ниобия обнаружены в многослойной системе $\mathrm{Cu}-\mathrm{Nb}$, полученной методом магнетронного напыления.

\section{4. Выводы}

B in situ сборных композитах $\mathrm{Cu}-18 \mathrm{Nb}$ большого сечения с истинной деформацией 10,2 и 12,5 формируются волокна ниобия ленточной формы, причем с ростом степени деформации максимальная толщина $\mathrm{Nb}$ лент уменьшается от $\sim 120$ до $\sim 70$ нм.

$\mathrm{B}$ ниобиевых волокнах композита $\mathrm{Cu}-18 \mathrm{Nb}(e=10,2$ и $e=12,5)$ обнаружены как микронапряжения, так и макронапряжения, возрастающие с ростом степени деформации. Макронапряжения приводят к искажению решетки ниобия - растягиванию межплоскостных расстояний $(110)_{\mathrm{Nb}}$ вдоль ОД и сжатию их в направлении, перпендикулярном ОД, причем с ростом степени деформации эти искажения заметно увеличиваются.

В медной матрице композита $\mathrm{Cu}-18 \mathrm{Nb}$ также обнаружен высокий уровень микронапряжений, возрастающий с ростом степени деформации. Кроме того, присутствуют и небольшие макронапряжения, которые приводят к искажениям решетки меди, хотя последние на порядок меньше, чем в ниобии.

\section{Благодарность}

Работа выполнена на оборудовании ЦКП ИФМ УрО РАН, в рамках государственного задания ФАНО России (тема «Давление», № АААA-А18-118020190104-3), при частичной поддержке УрО РАН (проект № 18-10-2-24).

\section{Список литературы}

1. Heringhaus F., Raabe D. Recent Advances in the Manufacturing of Copper-Base Composites // J. Mater. Proc. Technol. - 1996. - Vol. 59. - P. 367-372. - DOI: 10.1016/09240136(95)02179-5.

2. Raabe D., Mattissen D. Microstructure and mechanical properties of a cast and wire-drawn ternary $\mathrm{Cu}-\mathrm{Ag}-\mathrm{Nb}$ in situ composite // Acta Mater. - 1998. - Vol. 46, no. 16. - P. 5973-5984. DOI: 10.1016/S1359-6454(98)00218-3. 
3. The fabrication, properties and microstructure of $\mathrm{Cu}-\mathrm{Ag}$ and $\mathrm{Cu}-\mathrm{Nb}$ composites / $\mathrm{K}$. Han, J. D. Embury, J. R. Sims, L. J. Campbell, H.-J. Schneider-Muntau, V. I. Pantsyrnyi // Mater. Sci. Eng. - 1999. - Vol. A267. - P. 99-114. - DOI: 10.1016/S0921-5093(99)00025-8.

4. Internal stresses in cold-deformed $\mathrm{Cu}-\mathrm{Ag}$ and $\mathrm{Cu}-\mathrm{Nb}$ wires / $\mathrm{K}$. Han, A. C. Lawson, J. T. Wood, J. D. Embury, R. B. Von Dreele, J. W. Richardson // Phil. Mag. - 2004. - Vol. 84, no. 24. - P. 2579-2593. - DOI: 10.1080/14786430410001689981.

5. Influence of $\mathrm{Fe}$ addition on microstructure and properties of $\mathrm{Cu}-\mathrm{Ag}$ composite / X. Zuo, J. Zhu, B. An, K. Han, R. Li, E. Wang // Met. Mater. Int. - 2017. - Vol. 23, no. 5. - P. 974-983. DOI: $10.1007 / \mathrm{s} 12540-017-6656-2$.

6. Bevk J., Harbison J. P., Bell J. L. Anomalous increase in strength of in situ formed Cu$\mathrm{Nb}$ multifilamentary composites // J. Appl. Phys. - 1978. - Vol. 49, no. 12. - P. 6031-6038. DOI: $10.1063 / 1.324573$.

7. Spitzig W. A., Krotz P. D. A comparison of the strength and microstructure of heavily cold worked $\mathrm{Cu}-20 \% \mathrm{Nb}$ composite formed by different melting procedures // Scripta Metall. - 1987. Vol. 21, no. 8. - P. 1143-1146. - DOI: 10.1016/0036-9748(87)90266-3.

8. High strength, high conductivity $\mathrm{Cu}-\mathrm{Nb}$ based conductors with nanoscaled microstructure / A. Shikov, V. Pantsyrnyi, A. Vorobieva, N. Khlebova, A. Silaev // Phys. C. - 2001. - Vol. 354, no. 1-4. - P. 410-414. - DOI: 10.1016/S0921-4534(01)00109-5.

9. Pantsyrnyi V. Status and perspectives for microcomposite winding materials for high field pulsed magnets // IEEE Trans. Appl. Supercond. - 2002. - Vol. 12. - P.1189-1194. DOI: $10.1109 /$ TASC.2002.1018614.

10. Stability Aspects of the High Strength High Conductivity Microcomposite $\mathrm{Cu}-\mathrm{Nb}$ Wires Properties / V. Pantsyrny, A. Shikov, A. Vorobieva, N. Khlebova, N. Kozlenkova, I. Potapenko, M. Polikarpova // IEEE Trans. Appl. Supercond. - 2006. - Vol. 16, no. 2. - P. 1656-1659. DOI: $10.1109 /$ TASC.2006.870554.

11. Microstructure in $\mathrm{Cu}-\mathrm{Nb}$ microcomposites / Y. Leprince-Wang, K. Han, Y. Huang, K. YuZhang // Mater. Sci. Eng. A. - 2003. - Vol. A351. - P. 214-223. - DOI: 10.1016/S09215093(02)00855-9.

12. Transport characteristics of a $\mathrm{CuNb} / \mathrm{Nb} 3 \mathrm{Sn}$ superconducting coil fabricated using a react and wind method / G. Nishijima, S. Awaji, K. Watanabe, K. Miyoshi, A. Kimura // Supercond. Sci. Technol. - 2003. - Vol. 16. - P. 1082-1085. - DOI: 10.1088/0953-2048/16/9/320.

13. Grain structure and irreversibility line of a bronze route $\mathrm{CuNb}$ reinforced $\mathrm{Nb} 3 \mathrm{Sn}$ multifilamentary wire / M. J .R. Sandim, D. Stamopoulos, E. Aristomenopoulou, S. Zaefferer, D. Raabe, S. Awaji, K. Watanabe // Phys. Procedia. - 2012. - Vol. 36. - P. 1504-1509. DOI: 10.1016/j.phpro.2012.06.122.

14. Prebending Effect for Mechanical and Superconducting Properties of Nb-Rod-Processed $\mathrm{Cu}-\mathrm{Nb}$ Internal-Reinforced Nb3Sn Wires / H. Oguro, S. Awaji, K. Watanabe, M. Sugimoto, H. Tsubouchi // IEEE Transact. Appl. Supercond. - 2014. - Vol. 24, no. 3. - P. 8401004 (4). DOI: $10.1109 /$ tasc.2013.2292507.

15. Development of $\mathrm{Nb}-\mathrm{Rod}-\mathrm{Method} \mathrm{Cu}-\mathrm{Nb}$ Reinforced Nb3Sn Rutherford Cables for Reactand-Wind Processed Wide-Bore High Magnetic Field Coils / M. Sugimoto, H. Tsubouchi, S. Endoh, A. Takagi, K. Watanabe, S. Awaji, H. Oguro // Transact. Appl. Supercond. - 2015. Vol. 25. - P. 6000605. - DOI: 10.1109/TASC.2014.2381459.

16. Microstructure and texture of in situ heavily drawn $\mathrm{Cu}-\mathrm{Nb}$ composites / E. N. Popova, V. V. Popov, L. A. Rodionova, S. V. Sudareva, E. P. Romanova, A. E. Vorobyova, N. E. Hlebova, V. I. Pantsyrnyi, A. K. Shikov // Textures and Microstructures. - 2000. Vol. 34. - P. 263-277. - DOI: 10.1155/TSM.34.263.

17. Effect of alloying and regimes of thermo-mechanical treatment on the structure and properties of $\mathrm{Cu}-\mathrm{Nb}$ composites / E. N. Popova, V.V. Popov, E. P. Romanov, L. A. Rodionova, S. V. Sudareva, N. E. Khlebova, V. I. Pantsyrnyi, A. K. Shikov, A. E. Vorob'eva // Phys. Met. Metallogr. - 2002. - Vol. 94, no. 1. - P. 73-81. 
18. Structure and Thermal Stability of High-Strength $\mathrm{Cu}-18 \mathrm{Nb}$ Composite Depending on the Degree of Deformation / I. L. Deryagina, E. N. Popova, E. G. Valova-Zaharevskaya, E. I. Patrakov // Phys. Met. Metallogr. - 2018. - Vol. 119, no. 1. - P. 92-102. - DOI: 10.1134/S0031918X18010088.

19. Microstructure and texture evolution of $\mathrm{Cu}-\mathrm{Nb}$ composite wires / L. Deng, X. Yang, K. Han, Y. Lu, M. Liang, Q. Liu // Mater. Character. - 2013. - Vol. 81. - P. 124-133. DOI: 10.1016/j.matchar.2013.04.013.

20. Hardness, electrical resistivity, and modeling of in situ $\mathrm{Cu}-\mathrm{Nb}$ microcomposites / L. Deng, K. Han, K. T. Hartwig, T. M. Siegrist, L. Dong, Z. Sun, X. Yang, Q. Liu // J. Alloys and Compounds. - 2014. - Vol. 602. - P. 331-338. - DOI: 10.1016/j.jallcom.2014.03.021.

21. Solid state amorphization in cold drawn $\mathrm{Cu} / \mathrm{Nb}$ wires / $\mathrm{X}$. Sauvage, L. Renaud, B. Deconihout, D. Blavette, D. Ping, H. Hono // Acta Mater. - 2001. - Vol. 49. - P. 389. DOI: $10.1016 /$ S1359-6454(00)00338-4.

22. Microstructural characterization of high strength and high conductivity nanocomposite wires / F. Dupouy, E. Snoeck, M. J. Casanove, C. Roucau, J. P. Peyrade, S. Askenazy // Scripta Mater. 1996. - Vol. 34, no. 7. - P. 1067-1073. - DOI: 10.1016/1359-6462(95)00632-X.

23. Microstructural studies of in situ produced filamentary $\mathrm{Cu} / \mathrm{Nb}$ wires / $\mathrm{E}$. Snoeck, F. Lecouturier, L. Thilly, M. J. Casanove, H. Rakoto, G. Coffe, S. Aske'nazy, J. P. Peyrade, C. Roucau, V. Pantsyrny, A. Shikov, A. Nikulin // Scripta Mater. - 1998. - Vol. 38, no. 11. P. 1643-1648. - DOI: 10.1016/S1359-6462(98)00080-3.

24. Hangen U., Raabe D. Modelling of the yield strength of a heavily wire drawn $\mathrm{Cu}-20 \% \mathrm{Nb}$ composite by use of a modified linear rule of mixtures // Acta Metall. Mater. - 1995. - Vol. 43, no. 11. - P. 4075-4082. - DOI: 10.1016/0956-7151(95)00079-B.

25. Microstructural analysis of in-situ copper-niobium composite wires / A. R. Pelton, F. C. Laabs, W. A. Spitzig, C. C. Chen // Ultramicroscopy. - 1987. - Vol. 22 (1-4). - P. 251-265. DOI: 10.1016/0304-3991(87)90069-6.

26. On the texture evolution in swaged Cu-based wires / P. T. Kalu, L. Brandao, F. Ortiz, Okechukwu Egungwu, Funmi Ige // Scripta Mater. - 1998. - Vol. 38, no. 12. - P. 1755-1761. - DOI: 10.1016/S1359-6462(98)00114-6.

27. Copper-niobium high-strength and high-conductivity winding wires for pulsed magnets / A. K. Shikov, V. I. Pantsyrnyi, A. E. Vorob'eva, S. V. Sud'ev, N. E. Khlebova, A. K. Silaev, N. A. Belyakov // Metal Science and Heat Treatment. - 2002. - Vol. 44. - P. 491-495. DOI: $10.1023 / \mathrm{A}: 1022504805662$.

28. Transmission electron microscopy investigation of the atomic structure of interfaces in nanoscale $\mathrm{Cu}-\mathrm{Nb}$ multilayers / K. Yu-Zhang, J. D. Embury, K. Han, A. Misra // Phil. Mag. 2008. - Vol. 88, no. 17. - P. 2559-2567. - DOI: 10.1080/14786430802380485. 